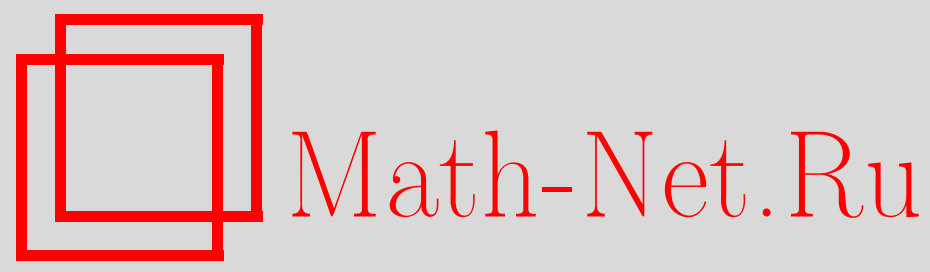

Е. Н. Шананина, Полиномиальные модели степени 5 и алгебры их автоморфизмов, Матем. заметки, 2004, том 75, выпуск 5, 757-772

DOI: https://doi.org/10.4213/mzm69

Использование Общероссийского математического портала Math-Net.Ru подразумевает, что вы прочитали и согласны с пользовательским соглашением http://www.mathnet.ru/rus/agreement

Параметры загрузки:

IP : 54.147 .182 .235

26 апреля 2023 г., 14:59:41 


\section{ПОЛИНОМИАЛЬНЫЕ МОДЕЛИ СТЕПЕНИ 5 \\ И АЛГЕБРЫ ИХ АВТОМОРФИЗМОВ}

\section{Е.Н. Шананина}

В вопросах классификации и изучения голоморфных автоморфизмов поверхностей часто оказьвается удобным перейти к рассмотрению касательной модельной поверхности. Этот метод хорошо разработан для поверхностей типа $(n, K)$ при $K \leqslant n^{2}$, для которых строится касательная квадрика - поверхность, заданная уравнениями степени 2, с рядом полезных свойств. В последние годы для поверхностей более высокой коразмерности были построены также касательные модельные поверхности степеней 3 и 4 с аналогичными свойствами. Однако на коразмерность при этом возникают новые ограничения. В данной работе этот метод применен для поверхностей еще более высокой коразмерности. Построены модельные поверхности пятой степени. Показано, что все основные полезные свойства модельной поверхности сохраняются, несмотря на ряд возникающих технических затруднений.

Библиограффия: 9 названий.

Введение. В изучении вещественных подмногообразий комплексного пространства и их голоморфных автоморфизмов большую роль играет касательная квадрика. (Этим вопросам было посвящено много работ; например, назовем [1]-[3]). Квадрика обладает рядом полезных свойств, делающих ее удобной для изучения биголоморфных отображений поверхностей.

1) Каждому порождающему ростку вещественно-аналитического подмногообразия комплексного пространства соответствует некоторая касательная квадрика.

2) Если квадрика невырождена в смысле [2], то группа ее голоморфных автоморфизмов конечномерна.

3) Квадрика однородна, т.е. группа ее автоморфизмов транзитивна.

4) Алгебра инфинитезимальных автоморфизмов невырожденной квадрики состоит из полей с полиномиальными коэффициентами, чья степень не превышает 2.

5) Группа ее биголоморфных автоморфизмов есть подгруппа групшы бирациональных преобразований объемлющего пространства. Существует оценка на степени числителей и знаменателей. Она зависит от размерности комплексной касательной и коразмерности квадрики (и исходного ростка). А именно, степень не превьшает $4(n+K)$, где $K$ - коразмерность, а $n$ - размерность комплексной части касательного пространства; поверхность является $C R$-многообразием типа $(n, K)$.

6) Квадрика обладает следуюшим экстремальным свойством: размерность групшы биголоморфных автоморфизмов произвольной поверхности мажорируется размерностью группы биголоморфных автоморфизмов ее касательной квадрики.

7) Если две квадрики биголоморфно эквивалентны, то они линейно эквивалентны. 
8) Квадрика обладает естественной структурой грушы Ли.

Поэтому, если многообразию соответствует невырожденная квадрика, исследование его свойств сильно упрощается. Но условие невырожденности квадрики накладьвает ограничение на коразмерность ростка: $K \leqslant n^{2}$. Хотелось бы иметь модельную поверхность, аналогичную квадрике, и в случае большей коразмерности. В работе [4] построены такие модельные поверхности степеней 3 и 4, задающиеся в некоторых координатах уравнениями вида

$$
\operatorname{Im} w=F(z, \bar{z})
$$

где $F$ - вектор-многочлен степени 3 при $n^{2}<K \leqslant n^{2}+n^{2}(n+1)$ и степени 4 при $n^{2}+n^{2}(n+1)<K \leqslant n^{2}+n^{2}(n+1)+n^{2}(n+1)(7 n+11) / 12$, однако здесь снова возникают ограничения на коразмерность. Там же предложены и модели $C R$-многообразий более высоких степеней, правда, без вьполнения свойства 1, т.е. универсальности. Как показано в работе автора [5], уже для поверхностей типа $(1,7)$ (минимальная ситуация, в которой не хватает четвертой степени, и появляется степень 5) не удается в общем случае построить модель такого вида. Однако, если разрешить правой части зависеть от $\operatorname{Re} w$, мы снова можем построить модельную поверхность с требуемыми свойствами. Наличие $\operatorname{Re} w$ в уравнениях модели несколько меняет доказательство конечномерности алгебры инфинитезимальных автоморфизмов, так как, например, становится невозможным применение теоремы об экспоненциальном представлении [6], однако все свойства, аналогичные вьшеперечисленньм, продолжают вьполняться. В данной работе снова изучается ситуация, в которой в уравнениях модели появляется степень 5 , однако на этот раз в случае произвольной размерности комплексной касательной, т.е. $C R$-многообразия типа $\left(n, k_{2}+k_{3}+k_{4}+k\right)$. (Здесь $k_{j}$ - размерность пространства вещественных однородных многочленов степени $j$ от $z, \bar{z}$, чье разложение по бистепеням не содержит компонент $(j, 0)$ и $(0, j) ; k \leqslant k_{5}+k_{2} k_{3}$; как подсчитано в [4], $k_{2}=n^{2}, k_{3}=n^{2}(n+1)$, $k_{4}=n^{2}(n+1)(7 n+11) / 12, k_{5}=n^{2}(n+1)(n+2)(3 n+5)$.) В п. 1 строятся модели степени 5. Невырожденность поверхности определим аналогично тому, как это сделано в работах [4], [5], [7]: после некоторых замен координат потребуем максимального ранга на уровне 4-струи и линейной независимости на уровне 5-струи.

Это условие можно перефомулировать и в инвариантных терминах. Нужно потребовать принадлежности к минимально возможному при данной коразмерности (конечному) типу по Блуму-Грэхму [8], т.е. к типу $\left(l_{1}, l_{2}, \ldots, l_{K}\right)$, где $l_{j}=2$ при $j=1, \ldots, k_{2}$; $l_{j}=3$ при $j=k_{2}+1, \ldots, k_{2}+k_{3} ; l_{j}=4$ при $j=k_{2}+k_{3}+1, \ldots, k_{2}+k_{3}+k_{4} ; l_{j}=5$ при $j=k_{2}+k_{3}+k_{4}+1, \ldots, k_{2}+k_{3}+k_{4}+k=K$. В п. 2 доказьвается конечномерность алгебры инфинитезимальных автоморфизмов модели и полиномиальность касательных голоморфных векторных полей, выводятся оценки на степень полей и размерность алгебры. В п. 3 проверяются бирациональность автоморфизмов, экстремальное свойство модели, линейная эквивалентность биголоморфно эквивалентных моделей, а также свойство однородности. Таким образом, доказывается следующая основная

ТеоремА. Невырохсденая модельная поверхность пятой степени типа ( $n$, $\left.k_{2}+k_{3}+k_{4}+k\right), k \leqslant k_{5}+k_{2} k_{3}$, является “хорошей” модельной поверхностью, а именно:

1) группа автоморфизмов невырожденной модели конечномерна (см. предложение 2.1);

2) всякому невырожденному порождающему ростку (или, что то же самое, ростку типа $\left(l_{1}, l_{2}, \ldots, l_{K}\right)$ по Блуму-Грәхму, где $l_{j}=2$ при $j=1, \ldots, k_{2}$, 
$l_{j}=3 n p u j=k_{2}+1, \ldots, k_{2}+k_{3}, l_{j}=4 n p u j=k_{2}+k_{3}+1, \ldots, k_{2}+k_{3}+k_{4}$, $l_{j}=5$ при $\left.j=k_{2}+k_{3}+k_{4}+1, \ldots, k_{2}+k_{3}+k_{4}+k=K\right)$ соответствует касательная модельная поверность пятой степени (см. в п. 1 определение 2 касательной модельной поверхности и предложение 1);

3) модельная поверхность пятой степени однородна, т.е. группа ее автоморфизмов транзитивна (см. предложение 3.2 );

4) алгебра инфинитезимальных автоморфизмов невырожденной модельной поверхности состоит из полей с полиномиальными коэффициентами, чья степень не превышает $k+5$ (см. предложение 2.2);

5) группа ее автоморфизмов есть подгруппа группь бирациональных преобразований обтемлющего пространства; существует оценка на степени числителей и знаменателей, зависящая от размерности комплексной касательной и коразмерности данной модельной поверхности (см. предложение 3.4$)$;

6) невырохсденная модельная поверхность обладает следующим әкстремальным свойством: размерность группы произвольной поверхности мажсорируется размерностью группь ее касательной модельной поверхности (см. предложение 3.1 );

7) если модельные поверхности биголоморфно эквивалентны, то они линейно әквивалентны (см. предложение 3.5);

8) модельная поверхность обладает естественной структурой әруппь Ли (см. предложение 3.3).

ЗАмЕчАнИЕ. Конечномерность алгебры можно было бы получить и с помощью теоремы 2 из [9].

Действительно, требование конечного типа по Блуму-Грэхму вьполнено, а требование конечной невырожденности при нашем типе вьполняется автоматически (уже форма Леви невырождена, а значит, у нас имеется 1-невырожденность). Однако чтобы получить полиномиальность полей, приходится заняться непосредственным вычислением алгебры.

1. Построение модели. Рассмотрим в пространстве $\mathbb{C}^{n+k_{2}+k_{3}+k_{4}+k}$ c координатами $z \in \mathbb{C}^{n}, w_{j}=x_{j}+i y_{j} \in \mathbb{C}^{k_{j}}, j=1, \ldots, 4 ; w_{5}=x_{5}+i y_{5} \in \mathbb{C}^{k}-$ порождающий росток вещественно-аналитического $C R$-многообразия типа $(n, K), K=k_{2}+k_{3}+k_{4}+k$ (т.е. с $n$-мерной комплексной касательной), заданный в нуле уравнениями

$$
y=F(z, \bar{z}, x)
$$

Применив, если нужно, аффинную замену координат, будем дальше считать, что функция $F$ обрашается в начале координат в нуль вместе со своими первьми производными.

Далее везде для записи степени будем использовать сокращенные обозначения. Так, запись $z^{\alpha_{1}} \bar{z}^{\alpha_{2}} x_{2}^{\beta_{2}} x_{3}^{\beta_{3}} x_{4}^{\beta_{4}} x_{5}^{\beta_{5}}$ будет означать полилинейную форму степени $\alpha_{1}$ по $z_{1}, \ldots$, $z_{n}$, степени $\alpha_{2}$ по $\bar{z}_{1}, \ldots, \bar{z}_{n}$, степеней $\beta_{j}$ по переменным соответствующих групп $x_{j}$, $j=2, \ldots, 5$.

Введем градуировку, задавая веса переменных так: $[z]=[\bar{z}]=1,\left[w_{j}\right]=\left[x_{j}\right]=j$, $j=2, \ldots, 5$.

Так как линейных членов $F$ не содержит, компоненты веса 1 нет. Рассмотрим теперь вторую весовую компоненту $F_{2}$. Она содержит члены, зависяшие только от $z, \bar{z}$ 
вида $z^{2}, \bar{z}^{2}$ и $z \bar{z}$. Квадратичной заменой $w \mapsto w+C_{2}(z, z)$, где $C_{2}-$ некоторая квадратичная форма от $z$, уберем члены при $z^{2}$ и $\bar{z}^{2}$. Оставшуюся (эрмитову) компоненту обозначим $F_{11}(z \bar{z})$. Так как $y \quad K$-мерен, это $K$-компонентная эрмитова вектор-форма. Для невырожденности потребуем от координатных компонент этой формы, чтобы их вещественная линейная оболочка составляла все пространство эрмитовых форм от $z, \bar{z}$. Поскольку это пространство $k_{2}$-мерно, а размерность $y_{2}$ в нашем случае также равна $k_{2}$, то, перенумеровьвая при необходимости координаты $y$, добьемся того, чтобы базис в прстранстве эрмитовьх форм образовывали именно те координатные компоненты, которые относятся к $y_{2}$. Теперь, вычитая из уравнений для $y_{3}, y_{4}, y_{5}$ линейные комбинации уравнений для $y_{2}$ (т.е. совершая линейную замену $w_{j} \mapsto w_{j}+r_{j} w_{2}, j=3,4,5$ ), уберем вторую весовую компоненту из уравнений для $y_{3}, y_{4}, y_{5}$.

Дальше в уравнениях для $y_{3}, y_{4}, y_{5}$ преобразуем компоненту веса 3 . Здесь у нас есть члены видов $z^{3}, \bar{z}^{3}, z^{2} \bar{z}, z \bar{z}^{2}, z x_{2}$ и $\bar{z} x_{2}$. От компонент $z^{3}, \bar{z}^{3}$ избавимся заменой $w \mapsto$ $w+C_{3}(z, z, z)$, где $C_{3}$ - некоторая кубическая форма от $z$; от $z x_{2}$ и $\bar{z} x_{2}-$ заменой $w \mapsto$ $w+D_{1}\left(z, w_{2}\right)$, где $D_{1}$ - билинейная форма от $z, w_{2}$. Теперь в весе 3 остаются только члены видов $z^{2} \bar{z}$ и $z \bar{z}^{2}$, которые будем обозначать через $F_{21}\left(z^{2} \bar{z}\right)$ и $F_{12}\left(z \bar{z}^{2}\right)$ соответственно (здесь $F_{21}$ и $F_{12}-\left(K-k_{2}\right)$-мерные полилинейные формы от $\left.z, \bar{z}\right)$. Для невырожденности снова потребуем, чтобы вешественная линейная оболочка их координатных компонент составляла все пространство вешественных однородных многочленов степени 3 от $z, \bar{z}$, чье разложение по бистепеням не содержит компонент $z^{3}$ и $\bar{z}^{3}$. Снова, перенумеровывая при необходимости координаты $y$, добьемся того, чтобы базис образовывали именно те координатные компоненты, которые относятся к $y_{3}$. Вычитая из уравнений для $y_{4}, y_{5}$ линейные комбинации уравнений для $y_{3}$ (т.е. совершая линейную замену $w_{j} \mapsto w_{j}+s w_{3}$, $j=4,5)$, уберем компоненту веса 3 из уравнений для $y_{4}, y_{5}$.

Преобразуем теперь компоненту веса 4 в уравнениях для $y_{4}$ и $y_{5}$. Здесь есть $z^{4}, \bar{z}^{4}$, $z^{3} \bar{z}, z \bar{z}^{3}, z^{2} \bar{z}^{2}, z^{2} x_{2}, \bar{z}^{2} x_{2}, z \bar{z} x_{2}, x_{2}^{2}, z x_{3}$ и $\bar{z} x_{3}$. От $z^{4}, \bar{z}^{4}$ избавимся заменой $w \mapsto w+$ $C_{4}(z, z, z, z)$, где $C_{4}$ - некоторая форма четвертой степени от $z$; от $z^{2} x_{2}$ и $\bar{z}^{2} x_{2}$ - заменой $w \mapsto w+D_{2}\left(z, z, w_{2}\right)$, где $D_{2}$ - некоторая полилинейная форма второй степени по $z$ и первой по $w_{2}$; от $z x_{3}$ и $\bar{z} x_{3}-$ заменой $w \mapsto w+D_{3}\left(z, w_{3}\right)$, где $D_{3}$ - некоторая билинейная форма от $z$ и $w_{3}$. От членов $z \bar{z} x_{2}$ и $x_{2}^{2}$ избавимся с помощью замены $w \mapsto w+D_{4}\left(w_{2}, w_{2}\right)$, где $D_{4}$ - некоторая квадратичная форма от $w_{2}$. Остаются только члены видов $z^{3} \bar{z}, z \bar{z}^{3}$ и $z^{2} \bar{z}^{2}$, которые будем обозначать через $F_{31}\left(z^{3} \bar{z}\right), F_{13}\left(z \bar{z}^{3}\right)$ и $F_{22}\left(z^{2} \bar{z}^{2}\right)$ соответственно (здесь $F_{31}, F_{13}$ и $F_{23}-\left(K-k_{2}-k_{3}\right)$-мерные полилинейные формы от $\left.z, \bar{z}\right)$. Опять потребуем для невырожденности, чтобы вещественная линейная оболочка их координатных компонент составляла все пространство вещественных однородных многочленов степени 4 от $z, \bar{z}$, чье разложение по бистепеням не содержит компонент $z^{4}$ и $\bar{z}^{4}$. И опять, перенумеровывая при необходимости координаты $y$, добьемся того, чтобы базис образовьвали именно те координатные компоненты, которые относятся к $y_{4}$. Затем вычитаем из уравнений для $y_{5}$ линейную комбинацию уравнений для $y_{4}$, т.е. делаем замену $w_{5} \mapsto w_{5}+s w_{3}$.

Теперь разложение по весам уравнений для $y_{5}$ начинается с пятой компоненты. Преобразуем ее тоже. В весе 5 у нас есть члены следующих видов: $z^{5}, \bar{z}^{5}, z^{4} \bar{z}, z \bar{z}^{4}, z^{3} \bar{z}^{2}$, $z^{2} \bar{z}^{3}, z^{2} \bar{z} x_{2}, z \bar{z}^{2} x_{2}, z x_{2}^{2}, \bar{z} x_{2}^{2}, z^{2} x_{3}, \bar{z}^{2} x_{3}, z \bar{z} x_{3}, z^{3} x_{2}, \bar{z}^{3} x_{2}, x_{2} x_{3}, z x_{4}$ и $\bar{z} x_{4}$. От $z^{5}$ и $\bar{z}^{5}$ избавимся заменой $w_{5} \mapsto w_{5}+C_{5}(z, z, z, z, z)$, где $C_{5}$ - некоторая форма пятой степени от $z$; от $z^{3} x_{2}$ и $\bar{z}^{3} x_{2}-$ заменой $w_{5} \mapsto w_{5}+D_{5}\left(z, z, z, w_{2}\right)$, где $D_{5}$ - некоторая полилинейная форма третьей степени по $z$ и первой по $w_{2}$; от $z^{2} x_{3}$ и $\bar{z}^{2} x_{3}$ - заменой $w_{5} \mapsto w_{5}+D_{6}\left(z, z, w_{3}\right)$, где $D_{6}$ - некоторая полилинейная форма второй степени по $z$, 
первой по $w_{3}$; от $z x_{2}^{2}$ и $\bar{z} x_{2}^{2}-$ заменой $w_{5} \mapsto w_{5}+D_{7}\left(z, w_{2}, w_{2}\right)$, где $D_{7}$ - некоторая полилинейная форма первой степени по $z$ и второй по $w_{2}$; от $z x_{4}$ и $\bar{z} x_{4}-$ заменой $w_{5} \mapsto w_{5}+$ $D_{8}\left(z, w_{4}\right)$, где $D_{5}$ - некоторая билинейная форма от $z$ и $w_{4}$. От членов $z \bar{z} x_{3}, x_{2} x_{3}$ избавимся заменой $w_{5} \mapsto w_{5}+D_{9}\left(w_{2}, w_{3}\right)$, где $D_{9}$ - некоторая билинейная форма от $w_{2}$ и $w_{3}$. Теперь для невырожденности потребуем линейной независимости компонент веса 5 для $y_{5}$.

Итак, в весе 5 остались члены видов $z^{4} \bar{z}, z \bar{z}^{4}, z^{3} \bar{z}^{2}, z^{2} \bar{z}^{3}, z^{2} \bar{z} x_{2}$ и $z \bar{z}^{2} x_{2}$. Обозначим их соответственно через $F_{41}\left(z^{4} \bar{z}\right), F_{14}\left(z \bar{z}^{4}\right), F_{32}\left(z^{3} \bar{z}^{2}\right), F_{23}\left(z^{2} \bar{z}^{3}\right), F_{212}\left(x_{2} z^{2} \bar{z}\right)$ и $F_{122}\left(x_{2} z \bar{z}^{2}\right)$. Это $k$-мерные полилинейные формы. По условию невырожденности координатные компоненты суммы $F_{41}+F_{14}+F_{32}+F_{23}+F_{212}+F_{122}$ линейно независимы как многочлены от $z, \bar{z}, x$. Рассмотрим еще сумму $F_{41}+F_{14}+F_{32}+F_{23}$ (ее компоненты могут и не оказаться линейно независимьми; например, они точно не будут линейно независимыми, если $k>k_{5}$ ). Рассмотрим вещественную линейную оболочку кординатных компонент этой суммы. Выделим в ней базис, состоящий из некоторого набора этих координатных компонент (соответствующие этим выбранньм компонентам переменные $x_{5}, y_{5}, w_{5}$ далее будем назьвать “жсестким $u$ ”). Теперь из оставшихся уравнений для $y_{5}$ (их будем называть “нежесткими”) вычтем линейную комбинацию уравнений для "жестких" $y_{5}$ (такую, чтобы в этих "нежестких" уравнениях совсем избавиться от членов видов $z^{4} \bar{z}, z \bar{z}^{4}, z^{3} \bar{z}^{2}$ и $z^{2} \bar{z}^{3}$ ). Заметим, что теперь в "нежестких" уравнениях линейно независимы координатные компоненты $F_{212}+F_{122}$.

Преобразования завершены, дадим теперь

ОПРЕДЕЛЕНИЕ 1 . Пусть в пространстве $\mathbb{C}^{n+k_{2}+k_{3}+k_{4}+k}$ с координатами $z \in \mathbb{C}^{n}$, $w_{j}=x_{j}+i y_{j} \in \mathbb{C}^{k_{j}}, j=1, \ldots, 4, w_{5}=x_{5}+i y_{5} \in \mathbb{C}^{k}$ задан порождающий росток вещественно-аналитического $C R$-многообразия типа $(n, K), K=k_{2}+k_{3}+k_{4}+k$, в окрестности нуля определяемьй уравнениями

$$
y=F(z, \bar{z}, x) \text {. }
$$

Выделим в этих уравнениях компоненты веса $\leqslant 5$ и обозначим их через $\Phi(z, \bar{z}, x)$. Получим

$$
y=\Phi(z, \bar{z}, x)+\cdots .
$$

Пусть после описанных в этом пункте преобразований координатные компоненты $\Phi(z, \bar{z}$, $x)$ линейно независимы. Тогда будем называть это вещественно-аналитическое $C R$-многообразие типа $\left(n, k_{2}+k_{3}+k_{4}+k\right)$ невырожденным.

ОпРЕДЕЛЕниЕ 2. В условиях и обозначениях определения 1 поверхность, заданную уравнениями

$$
y=\Phi(z, \bar{z}, x)
$$

будем назьвать (невырожсденой) касательной модельной поверхностью для нашего ростка.

ЗАмЕЧАнИЕ. Условие линейной независимости форм веса 5 для $y_{5}$ (традиционное для модельньх поверхностей условие невырожденности) в нашем случае (когда $l_{j}=2$ при $j=1, \ldots, k_{2} ; l_{j}=3$ при $j=k_{2}+1, \ldots, k_{2}+k_{3} ; l_{j}=4$ при $j=k_{2}+k_{3}+1, \ldots, k_{2}+$ $k_{3}+k_{4}$, т.е. формы для $y$ степеней $2,3,4$ образуют базис в соответствующих пространствах) равносильно принадлежности поверхности к типу $\left(l_{1}, l_{2}, \ldots, l_{K}\right)$ по Блуму-Грэхму 
(т.е. тому, что также $l_{j}=5$ при $j=k_{2}+k_{3}+k_{4}+1, \ldots, k_{2}+k_{3}+k_{4}+k=K$ ). Действительно, если формы веса 5 оказываются линейно зависимы, это означает, что после некоторой линейной замены координат мы можем получить одну из координат $y_{j}$ без весовых компонент $2,3,4,5$, что означает принадлежность к большему типу, конечному или бесконечному. Если же формы линейно независимы, то принадлежность к типу $\left(l_{1}, l_{2}, \ldots, l_{K}\right)$ можно проверить, например, непосредственно выгисляя коммутаторы. (Или, зная наперед, что алгебра инфинитезимальных автоморфизмов конечномерна, а при доказательстве конечномерности мы будем пользоваться именно линейной независимостью, можно воспользоваться теоремой 3 работы [9] и получить конечность типа. А так как модельная поверхность задается однородными уравнениями веса 5 , этот тип не может быть больше, чем $\left(l_{1}, l_{2}, \ldots, l_{K}\right)$.) Заметим также, что требование линейной независимости (принадлежности к типу $\left.\left(l_{1}, l_{2}, \ldots, l_{K}\right)\right)$ накладывает очередное ограничение на коразмерность: $K \leqslant k_{2}+k_{3}+k_{4}+k_{5}+k_{2} k_{3}$.

Итак, доказано

ПРЕДЛОЖЕНИЕ 1. Пусть $M$ - росток порождающего вещественно-аналитического $C R$-многообразия типа $\left(n, k_{2}+k_{3}+k_{4}+k\right)$, невырожсденого в смысле определения 1. Тогда полиномиальной (степени не выше 5) заменой координат уравнения, задающие поверхность, приводятся к виду

$$
\begin{aligned}
& y_{2}=F_{11}(z \bar{z})+O(3) \\
& y_{3}=2 \operatorname{Re} F_{21}\left(z^{2} \bar{z}\right)+O(4), \\
& y_{4}=2 \operatorname{Re} F_{31}\left(z^{3} \bar{z}\right)+F_{22}\left(z^{2} \bar{z}^{2}\right)+O(5), \\
& y_{5}=2 \operatorname{Re}\left(F_{41}\left(z^{4} \bar{z}\right)+F_{32}\left(z^{3} \bar{z}^{2}\right)+F_{212}\left(x_{2} z^{2} \bar{z}\right)\right)+O(6) .
\end{aligned}
$$

Здесь $F_{p q}\left(z^{p} \bar{z}^{q}\right)$ обозначает полилинейную форму от $z=\left(z_{1}, \ldots, z_{n}\right), \bar{z}=\left(\bar{z}_{1}, \ldots, \bar{z}_{n}\right)$ степени $p$ по $z$, степени $q$ по $\bar{z} ; F_{p q r}\left(x_{j_{1}}^{l_{1}} \cdots x_{j_{m}}^{l_{m}} z^{p} \bar{z}^{q}\right)$ обозначает полилинейную форму от $z, \bar{z}$ степени р по $z$, степени $q$ по $\bar{z}$, степеней $l_{t}$ nо $x_{j_{t}}=\left(x_{j_{t}, 1}, \ldots, x_{j_{t}, k_{j_{t}}}\right)$ соответственно и степени по $x$, удовлетворяющей следующему ограничению на вес: $\left[x_{j_{1}}^{l_{1}} \cdots x_{j_{m}}^{l_{m}}\right] \leqslant r ; O(n)$ обозначает члены вес $\geqslant \geqslant n$.

Отбрасьвая в уравнениях старшие члены, получим касательную модельную поверхность $Q$.

2. Оценки для алгебры. Оценим размерности алгебры инфинитезимальных автоморфизмов ростка построенной невырожденной модельной поверхности в нуле aut $Q_{0}$ и подалгебры aut $_{0} Q_{0}$ инфинитезимальньх автоморфизмов, обращающихся в 0 в нуле. Пусть вещественное векторное поле

$$
X=2 \operatorname{Re}\left(f(z, w) \frac{\partial}{\partial z}+\sum_{j=2}^{5} g_{j}(z, w) \frac{\partial}{\partial w_{j}}\right)
$$

принадлежит алгебре aut $Q_{0}$. Это означает, что координатные компоненты вектор-функций $f, g_{j}$ голоморфны в окрестности нуля и их ограничения на $Q$ удовлетворяют соотношениям, определяющим касательную плоскость к $Q$ :

$$
\begin{aligned}
\operatorname{Re}\left(i g_{2}+2 F_{11}(f \bar{z})\right) & =0, \\
\operatorname{Re}\left(i g_{3}+2 F_{12}\left(f \bar{z}^{2}\right)+4 F_{21}(f z \bar{z})\right) & =0, \\
\operatorname{Re}\left(i g_{4}+2 F_{13}\left(f \bar{z}^{3}\right)+4 F_{22}\left(f z \bar{z}^{2}\right)+6 F_{31}\left(f z^{2} \bar{z}\right)\right) & =0, \\
\operatorname{Re}\left(i g_{5}+8 F_{41}\left(f z^{3} \bar{z}\right)+6 F_{32}\left(f z^{2} \bar{z}^{2}\right)+4 F_{212}\left(x_{2} f z \bar{z}\right)+2 F_{14}\left(f \bar{z}^{4}\right)\right. & \\
\left.+4 F_{23}\left(f z \bar{z}^{3}\right)+2 F_{122}\left(x_{2} f \bar{z}^{2}\right) F_{212}\left(g_{2} z^{2} \bar{z}\right)+F_{122}\left(g_{2} z \bar{z}^{2}\right)\right) & =0 .
\end{aligned}
$$


Будем использовать обозначения $\Delta_{p q} f=\partial_{x_{p+q}} f\left(F_{p q}\right), \Delta_{p q r} f=\partial_{x_{p+q+r}} f\left(F_{p q r}\right)$ (значение частного дифференциала на форме), а также разложения вида

$$
\varphi(z, w)=\varphi(z, x)+\sum_{j=1}^{K} \frac{\partial \varphi}{\partial x_{j}} i y_{j}+\cdots, \quad \varphi(z, x)=\sum_{q} \varphi_{(q)}\left(x, z^{q}\right)
$$

где $\varphi_{(q)}\left(x, z^{q}\right)$ - форма степени $q$ от $z_{1}, \ldots, z_{n}$ с коэффициентами, являюшимися функциями от $x$.

Будем выделять в выписанных соотношениях коэффициенты при $z^{l} \bar{z}^{m}$ (т.е. при мономах $z_{1}^{l_{1}} \ldots z_{n}^{l_{n}} \bar{z}_{1}^{m_{1}} \ldots \bar{z}_{n}^{m_{n}}$, где $\left.\sum_{j=1}^{n} l_{j}=l, \sum_{j=1}^{n} m_{j}=m\right)$.

Вначале рассмотрим случай $l=0, m=0$ (члены, не содержащие $z$ и $\bar{z}$ ):

$$
\operatorname{Im} g_{t(0)}=0, \quad t=2, \ldots, 5
$$

при $l=1, m=0$ (линейные по $z$ члены)

$$
\begin{aligned}
& g_{2(1)}=2 i F_{11}\left(z \bar{f}_{(0)}\right) \\
& g_{3(1)}=0 \\
& g_{4(1)}=0 \\
& g_{5(1)}=0
\end{aligned}
$$

при $1<l<5, m=0$ (коэффициенты при членах вида $\left.z^{l}\right)$

$$
\begin{aligned}
g_{2(l)} & =0 \\
\ldots \ldots \ldots \ldots \ldots \ldots \ldots & \ldots \ldots \ldots \\
g_{l+1(l)} & =2 i F_{l 1}\left(z^{l} \bar{f}_{(0)}\right), \\
g_{5(2)} & =2 i F_{212}\left(x_{2} z^{2} \bar{f}_{(0)}\right) ;
\end{aligned}
$$

при $l=1, m=1$ (коэффициенты при $z \bar{z})$

$$
\begin{aligned}
& \Delta_{11} g_{2(0)}=2 \operatorname{Re} F_{11}\left(f_{(1)} \bar{z}\right), \\
& \Delta_{11} g_{3(0)}=4 \operatorname{Re} F_{21}\left(f_{(0)} z \bar{z}\right), \\
& \Delta_{11} g_{4(0)}=0, \\
& \Delta_{11} g_{5(0)}=4 \operatorname{Re} F_{212}\left(x_{2} f_{(0)} z \bar{z}\right) ;
\end{aligned}
$$

при $l=2, m=1$ (коэффициенты при $\left.z^{2} \bar{z}\right)$

$$
\begin{aligned}
& \Delta_{21} g_{2(0)}+\Delta_{212} g_{2(0)}=2 F_{11}\left(f_{(2)} \bar{z}\right)-4 i F_{11}\left(z \Delta_{11} \bar{f}_{(0)}\right) \\
& \Delta_{21} g_{3(0)}+\Delta_{212} g_{3(0)}=4 F_{21}\left(f_{(1)} z \bar{z}\right)+2 F_{21}\left(z^{2} \bar{f}_{(1)}\right) \\
& \Delta_{21} g_{4(0)}+\Delta_{212} g_{4(0)}=6 F_{31}\left(f_{(0)} z^{2} \bar{z}\right)+4 F_{22}\left(z^{2} \bar{z} \bar{f}_{(0)}\right) \\
& \Delta_{21} g_{5(0)}+\Delta_{212} g_{5(0)}=2 F_{212}\left(\operatorname{Re} g_{2} z^{2} \bar{z}\right)+4 F_{212}\left(x_{2} f_{(1)} z \bar{z}\right)+2 F_{212}\left(x_{2} z^{2} \bar{f}_{(1)}\right)
\end{aligned}
$$


при $l=3, m=1$ (коэффициенты при $\left.z^{3} \bar{z}\right)$

$$
\begin{aligned}
\Delta_{31} g_{2(0)}= & 2 F_{11}\left(f_{(3)} \bar{z}\right)-4 i F_{11}\left(z \Delta_{21} \bar{f}_{(0)}\right)-4 i F_{11}\left(z \Delta_{212} \bar{f}_{(0)}\right), \\
\Delta_{31} g_{3(0)}= & 4 F_{21}\left(f_{(2)} z \bar{z}\right)-4 i F_{21}\left(z^{2} \Delta_{11} \bar{f}_{(0)}\right) \\
\Delta_{31} g_{4(0)}= & 6 F_{31}\left(f_{(1)} z^{2} \bar{z}\right)+2 F_{31}\left(z^{3} \bar{f}_{(1)}\right) \\
\Delta_{31} g_{5(0)}= & 8 F_{41}\left(f_{(0)} z^{3} \bar{z}\right)+4 F_{32}\left(z^{3} \bar{z} \bar{f}_{(0)}\right)+F_{212}\left(g_{2(1)} z^{2} \bar{z}\right) \\
& +4 F_{212}\left(x_{2} f_{(2)} z \bar{z}\right)-4 i F_{212}\left(x_{2} z^{2} \Delta_{11} \bar{f}_{(0)}\right)
\end{aligned}
$$

при $l=4, m=1$ (коэффициенты при $\left.z^{4} \bar{z}\right)$

$$
\begin{aligned}
\Delta_{41} g_{2(0)}= & 2 F_{11}\left(f_{(4)} \bar{z}\right)-4 i F_{11}\left(z \Delta_{31} \bar{f}_{(0)}\right), \\
\Delta_{41} g_{3(0)}= & 4 F_{21}\left(f_{(3)} z \bar{z}\right)-4 i F_{21}\left(z^{2} \Delta_{21} \bar{f}_{(0)}\right)-4 i F_{21}\left(z^{2} \Delta_{212} \bar{f}_{(0)}\right), \\
\Delta_{41} g_{4(0)}= & 6 F_{31}\left(f_{(2)} z^{2} \bar{z}\right)-4 i F_{31}\left(z^{3} \Delta_{11} \bar{f}_{(0)}\right), \\
\Delta_{41} g_{5(0)}= & 8 F_{41}\left(f_{(1)} z^{3} \bar{z}\right)+2 F_{41}\left(z^{4} \bar{f}_{(1)}\right)+F_{212}\left(g_{2(2)} z^{2} \bar{z}\right) \\
& +4 F_{212}\left(x_{2} f_{(3)} z \bar{z}\right)-4 i F_{212}\left(x_{2} z^{2} \Delta_{21} \bar{f}_{(0)}\right)-4 i F_{212}\left(x_{2} z^{2} \Delta_{212} \bar{f}_{(0)}\right) ;
\end{aligned}
$$

при $l=5, m=1$ (коэффициенты при $z^{5} \bar{z}$ )

$$
\begin{aligned}
F_{11}\left(f_{(5)} \bar{z}\right)= & 2 i F_{11}\left(z \Delta_{41} \bar{f}_{(0)}\right), \\
2 F_{21}\left(f_{(4)} z \bar{z}\right)= & 2 i F_{21}\left(z^{2} \Delta_{31} \bar{f}_{(0)}\right), \\
3 F_{31}\left(f_{(3)} z^{2} \bar{z}\right)= & 2 i F_{31}\left(z^{3} \Delta_{21} \bar{f}_{(0)}\right)+2 i F_{31}\left(z^{3} \Delta_{212} \bar{f}_{(0)}\right), \\
4 F_{41}\left(f_{(2)} z \bar{z}\right)= & 2 i F_{41}\left(z^{4} \Delta_{11} \bar{f}_{(0)}\right)-F_{212}\left(g_{2(3)} z^{2} \bar{z}\right) \\
& -4 F_{212}\left(x_{2} f_{(4)} z \bar{z}\right)+2 i F_{212}\left(x_{2} z^{2} \Delta_{31} \bar{f}_{(0)}\right) ;
\end{aligned}
$$

при $l=6, m=1$ (коэффициенты при $z^{6} \bar{z}$ )

$$
\begin{aligned}
F_{11}\left(f_{(6)} \bar{z}\right)= & 0 \\
2 F_{21}\left(f_{(5)} z \bar{z}\right)= & 2 i F_{21}\left(z^{2} \Delta_{41} \bar{f}_{(0)}\right), \\
3 F_{31}\left(f_{(4)} z^{2} \bar{z}\right)= & 2 i F_{31}\left(z^{3} \Delta_{31} \bar{f}_{(0)}\right), \\
4 F_{41}\left(f_{(3)} z^{3} \bar{z}\right)= & 2 i F_{41}\left(z^{4} \Delta_{21} \bar{f}_{(0)}\right)+2 i F_{41}\left(z^{4} \Delta_{212} \bar{f}_{(0)}\right)-F_{212}\left(g_{2(4)} z^{2} \bar{z}\right) \\
& -4 F_{212}\left(x_{2} f_{(5)} z \bar{z}\right)+2 i F_{212}\left(x_{2} z^{2} \Delta_{41} \bar{f}_{(0)}\right) ;
\end{aligned}
$$

при $l=7, m=1$ (коэффициенты при $\left.z^{7} \bar{z}\right)$

$$
\begin{aligned}
F_{11}\left(f_{(7)} \bar{z}\right) & =0 \\
F_{21}\left(f_{(6)} z \bar{z}\right) & =0 \\
3 F_{31}\left(f_{(5)} z^{2} \bar{z}\right) & =2 i F_{31}\left(z^{3} \Delta_{41} \bar{f}_{(0)}\right), \\
4 F_{41}\left(f_{(4)} z^{3} \bar{z}\right) & =2 i F_{41}\left(z^{4} \Delta_{31} \bar{f}_{(0)}\right)-F_{212}\left(g_{2(5)} z^{2} \bar{z}\right)-4 F_{212}\left(x_{2} f_{(6)} z \bar{z}\right) ;
\end{aligned}
$$

при $l=8, m=1$ (коэффициенты при $z^{8} \bar{z}$ )

$$
\begin{aligned}
F_{11}\left(f_{(8)} \bar{z}\right) & =0, \\
F_{21}\left(f_{(7)} z \bar{z}\right) & =0, \\
F_{31}\left(f_{(6)} z^{2} \bar{z}\right) & =0, \\
4 F_{41}\left(f_{(5)} z^{3} \bar{z}\right) & =2 i F_{41}\left(z^{4} \Delta_{41} \bar{f}_{(0)}\right)-F_{212}\left(g_{2(6)} z^{2} \bar{z}\right)-4 F_{212}\left(x_{2} f_{(7)} z \bar{z}\right) ;
\end{aligned}
$$


при $l \geqslant 6, m=1$ (коэффициенты при $\left.z^{l} \bar{z}\right)$

$$
F_{11}\left(f_{(l)} \bar{z}\right)=0
$$

откуда при $l \geqslant 6$ получаем $f_{(l)}=0$ (мы воспользовались здесь тем, что для невырожденной поверхности координатные компоненты $F_{11}$ составляют базис пространства $(1,1)$-форм).

Далее при нумерации вьписанных соотношений используем тройную индексацию. Запись $(p, q \mid r)$ будет означать соотношение, относяшееся к $z^{p} \bar{z}^{q}$ и $g_{r}$.

Помимо уже вьписанных нам понадобятся соотношения $(2,2 \mid 2),(2,2 \mid 3),(2,2 \mid 4),(2,2 \mid$ $5),(3,2 \mid 4),(3,2 \mid 5),(4,2 \mid 3),(5,2 \mid 3),(3,2 \mid 2)$ и $(3,2 \mid 3)$, которые выпишем позже.

Рассмотрим соотношение $(5,1 \mid 2)$. Оно должно вьполняться с каждой из координат формы $F_{11}$ и, следовательно, с любой эрмитовой формой от $z, \bar{z}$. Это равносильно тому, что оно выполняется для формы $z_{j} \bar{z}_{l}$ при любых $j, l$, т.е.

$$
f_{j(5)}\left(z^{5}\right) \bar{z}_{l}=2 i z_{j}\left(\Delta_{41} \bar{f}_{(0)}\right)_{l},
$$

откуда следует, что $f_{(5)}$ и $\Delta_{41} \bar{f}_{(0)}$ имеют следующий вид:

$$
f_{(5)}\left(z^{5}\right)=2 i \psi_{5}\left(z^{4}\right) z, \quad \Delta_{41} \bar{f}_{(0)}=\psi_{5}\left(z^{4}\right) \bar{z}
$$

где $\psi_{5}\left(z^{4}\right)$ - скалярньй множитель, являющийся однородным многочленом степени 4 от $z$. Теперь подставим полученные выражения в соотношение $(6,1 \mid 3)$. Получаем, что $\psi_{5}=0$, т.е. $f_{(5)}=0, \Delta_{41} \bar{f}_{(0)}=0$. Таким же образом из пар соотношений $(5,1 \mid 3)$ и $(6,1 \mid 4),(5,1 \mid 4)$ и $(6,1 \mid 5)$ получаем $f_{(4)}=0, f_{(3)}=0, \Delta_{31} \bar{f}_{(0)}=0,\left(\Delta_{21}+\Delta_{212}\right) \bar{f}_{(0)}=0$.

Теперь вьпишем (с учетом полученного) соотношения $(2,2 \mid 2)$ и $(2,2 \mid 3)$ :

$$
\begin{aligned}
& \Delta_{22} g_{2(0)}=F_{11}\left(z i \Delta_{11} \bar{f}_{(1)}\right)+F_{11}\left(i \Delta_{11} f_{(1)} \bar{z}\right), \\
& \Delta_{22} g_{3(0)}=F_{21}\left(z^{2} \bar{f}_{(2)}\right)+2 F_{21}\left(i \Delta_{11} f_{(0)} z \bar{z}\right)+2 F_{12}\left(z \bar{z} i \Delta_{11} \bar{f}_{(0)}\right)+F_{12}\left(f_{(2)} \bar{z}^{2}\right) .
\end{aligned}
$$

Обозначим теперь $f_{(0)}, f_{(1)}, f_{(2)}$ через $A(x), B(x) z, C(x) z^{2}$ соответственно, $g_{j(0)}$ через $G_{j}$. Пользуясь тем, что почти все $\Delta_{j k}$ и $\Delta_{j k l}$ попарно коммутируют (кроме пары $\Delta_{212}$ и $\Delta_{11}$, коммутатор которых тоже легко вычисляется), будем исключать из наших соотношений $G_{j}$. Применяя $\Delta_{21}+\Delta_{212}$ к $(1,1 \mid 4)$ и $\Delta_{11}$ к $(2,1 \mid 4)$, получим

$$
\frac{\partial G_{4}}{\partial x_{5}} \Delta_{11} F_{212}\left(x_{2} z^{2} \bar{z}\right)=\Delta_{11}\left(6 F_{31}\left(A z^{2} \bar{z}\right)+4 F_{22}\left(z^{2} \bar{z} \bar{A}\right)\right) .
$$

Применяя $\Delta_{31}$ к соотношениям $(1,1 \mid j)$ и $\Delta_{11}$ к $(3,1 \mid j)$, получим

$$
\begin{aligned}
\Delta_{31}\left(2 \operatorname{Re} F_{11}(B z \bar{z})\right) & =0 \\
\Delta_{31}\left(2 \operatorname{Re} 2 F_{21}(A z \bar{z})\right) & =\Delta_{11}\left(2 F_{21}\left(C z^{2} z \bar{z}\right)-4 i F_{21}\left(z^{2} \Delta_{11} \bar{A}\right),\right. \\
0 & =\Delta_{11}\left(6 F_{31}\left(B z z^{2} \bar{z}\right)+2 F_{31}\left(z^{3} \bar{B} z\right)\right) .
\end{aligned}
$$

Применяя $\Delta_{31}$ к соотношениям $(2,1 \mid j)$ и $\Delta_{21}+\Delta_{212}$ к $(3,1 \mid j)$, получим

$$
\begin{aligned}
\Delta_{31}\left(2 F_{11}\left(C z^{2} \bar{z}\right)-4 i F_{11}\left(z \Delta_{11} \bar{A}\right)\right) & =0, \\
\Delta_{31}\left(4 F_{21}(B z z \bar{z})+2 F_{21}\left(z^{2} \bar{B} z\right)\right) & =\left(\Delta_{21}+\Delta_{212}\right)\left(2 F_{21}\left(C z^{2} z \bar{z}\right)-4 i F_{21}\left(z^{2} \Delta_{11} \bar{A}\right)\right)=0,
\end{aligned}
$$

$$
\Delta_{31}\left(6 F_{31}\left(A z^{2} \bar{z}\right)+4 F_{22}\left(z^{2} \bar{z} \bar{A}\right)\right)=\left(\Delta_{21}+\Delta_{212}\right)\left(6 F_{31}\left(B z z^{2} \bar{z}\right)+2 F_{31}\left(z^{3} \bar{B} z\right)\right) .
$$


Применяя $\Delta_{22}$ к соотношениям $(2,1 \mid j)$ и $\Delta_{21}+\Delta_{212}$ к $(2,2 \mid j)$, получим

$$
\begin{aligned}
\Delta_{22}\left(2 F_{11}\left(C z^{2} \bar{z}\right)-4 i F_{11}\left(z \Delta_{11} \bar{A}\right)\right)= & 0, \\
\Delta_{22}\left(4 F_{21}(B z z \bar{z})+2 F_{21}\left(z^{2} \bar{B} z\right)\right)= & \left(\Delta_{21}+\Delta_{212}\right)\left(F_{21}\left(z^{2} C \bar{z}^{2}\right)+2 F_{21}\left(i \Delta_{11} A z \bar{z}\right)\right. \\
& \left.+2 F_{12}\left(z \bar{z} i \Delta_{11} \bar{A}\right)+F_{12}\left(C z^{2} \bar{z}^{2}\right)\right) .
\end{aligned}
$$

Применяя $\Delta_{41}$ к соотношениям $(2,1 \mid j)$ и $\Delta_{21}+\Delta_{212}$ к $(4,1 \mid j)$, получим

$$
\begin{aligned}
\Delta_{41}\left(2 F_{11}\left(C z^{2} \bar{z}\right)-4 i F_{11}\left(z \Delta_{11} \bar{A}\right)\right) & =0, \\
\Delta_{41}\left(4 F_{21}(B z z \bar{z})+2 F_{21}\left(z^{2} \bar{B} z\right)\right) & =0, \\
\Delta_{41}\left(6 F_{31}\left(A z^{2} \bar{z}\right)+F_{22}\left(z^{2} \bar{z} \bar{A}\right)\right) & =\left(\Delta_{21}+\Delta_{212}\right)\left(6 F_{31}\left(C z^{2} z^{2} \bar{z}\right)-4 i F_{31}\left(z^{3} \Delta_{11} \bar{A}\right)\right) \\
& =0 .
\end{aligned}
$$

Рассмотрим соотношение (4). Воспользовавшись $(1,1 \mid 4)$, получим, что тейлоровское разложение $A$ не содержит $x_{2}$ в степени вьше первой.

Теперь из (6) получаем $C_{x_{2}}^{\prime}=0$, из (7) $B_{x_{2}}^{\prime}=0$. Из соотношения (15) имеем $\left(\Delta_{21}+\Delta_{212}\right) C=0$, откуда с учетом $C_{x_{2}}^{\prime}=0$ получаем $C_{x_{3}}^{\prime}=0, \Delta_{212} C=0$. Из $(8)$ и (11) получаем $C_{x_{4}}^{\prime}=0$. Из соотношения (10) имеем $\left(\Delta_{21}+\Delta_{212}\right) B=0$, откуда с учетом $B_{x_{2}}^{\prime}=0$ получаем $B_{x_{3}}^{\prime}=0, \Delta_{212} B=0$. Из соотношений (9) и (12) получаем $B_{x_{4}}^{\prime}=0$. Из соотношения (13) имеем $\Delta_{41} C=0$. Из соотношения (14) получаем $\Delta_{41} B=0$.

Теперь с учетом всего полученного вьпишем $(4,2 \mid 3)$ :

$$
2 i F_{21}\left(z^{2} \Delta_{22} \bar{A}\right)=0 \text {. }
$$

Отсюда $\Delta_{22} A=0$, т.е. $A$ не зависит от переменных из группы $x_{4}$.

Аналогично, вьписав $(5,2 \mid 3)$ :

$$
2 i F_{21}\left(z^{2} \Delta_{32} \bar{A}\right)=0,
$$

получим $\Delta_{32} A=0$. Вспоминая, что также $\Delta_{41} A=0$, получаем, что $A$ не зависит от "жестких" переменных из группы $x_{5}$.

Итак, $A$ у нас может зависеть только от $x_{2}, x_{3}$ и “нежестких" переменных из группы $x_{5}$, причем $x_{2}$ не может входить в тейлоровское разложение в степени вьше первой.

Теперь посмотрим на соотношение

$$
\left(\Delta_{21}+\Delta_{212}\right) \bar{A}=0
$$

более внимательно, т.е. распишем его по степеням $z_{j_{1}} z_{j_{2}} \bar{z}_{j_{3}}$. Получим

$$
\sum_{q} \frac{\partial \bar{A}}{\partial x_{3, q}} F_{21, q}+\sum_{q, l} p_{q, l}\left(x_{2}\right) \frac{\partial \bar{A}}{\partial x_{5, l}} F_{21, q}=0 ;
$$

здесь $x_{3, q}, x_{5, l}, F_{21, q}$ обозначают конкретные координатные компоненты из соответствуюших груп переменных и полилинейных форм. Воспользовавшись тем, что $F_{21, q}$ образуют базис в пространстве форм бистепени $(2,1)$, получим для всех $q$

$$
\frac{\partial \bar{A}}{\partial x_{3, q}}+\sum_{l} p_{q, l}\left(x_{2}\right) \frac{\partial \bar{A}}{\partial x_{5, l}}=0,
$$

где $p_{q, l}$ - линейные функции от $x_{2}$.

Заметим, что, так как $A$ не зависит от "жестких" переменных из группы $x_{5}$, в эти уравнения не входят $p_{q l}\left(x_{2}\right)$, относящиеся к “жестким" $x_{5, l}$. Поэтому можно считать, что для входящих в $(16) p_{q l}\left(x_{2}\right)$ система полиномов от $x_{2}, x_{3}\left\{\sum_{q} p_{q l}\left(x_{2}\right) x_{3, q}\right\}_{l}$ линейно независима. 
Лемма. Пусть $A\left(x_{2}, x_{3}, x_{5}\right)$ - вешественно-аналитическая функиия, причем тейлоровское разложение $A$ не содержит $x_{2}$ в степени выше первой. Пусть при всех $q$ выполняется условие (16). Пусть также система $\left\{\sum_{q} p_{q l}\left(x_{2}\right) x_{3, q}\right\}_{l}$ линейно независима. Тогда $A$ можсно представить в виде

$$
A=\varphi_{0}\left(x_{5}-p\left(x_{2}, x_{3}\right)\right)+\sum_{q=1}^{k} x_{2, q} \varphi_{q}\left(x_{5}-p\left(x_{2}, x_{3}\right)\right)
$$

где $p\left(x_{2}, x_{3}\right)$ - билинейная вектор-функция от $x_{2}, x_{3} ; \varphi_{q}, q=0, \ldots, k,-$ многочлень степени, не превышающей $k$. Таким образом, $A\left(x_{2}, x_{3}, x_{5}\right)$ является многочленом степени не выше $2 k+1$.

ДокАЗАтЕльство. Рассмотрим $\bar{A}$ как функцию от $x_{3}, x_{5}$ с переменными из группы $x_{2}$ в качестве параметров, т.е. используем разложение

$$
\bar{A}=\sum_{r s} c_{r s}\left(x_{2}\right) x_{3}^{r} x_{5}^{s} .
$$

Сделаем замену $x_{3, q}=\xi_{q}, x_{5, l}=\eta_{l}+\sum_{q} p_{q l}\left(x_{2}\right) \xi_{q}$. Тогда соотношения (16) примут вид

$$
\frac{\partial \bar{A}}{\partial \xi_{q}}=0
$$

т.е. $\bar{A}=\bar{A}\left(\eta_{1}, \ldots, \eta_{k}\right)$. Таким образом,

$$
\bar{A}=\sum_{r_{1}, \ldots, r_{k}} C_{r_{1}, \ldots, r_{k}}\left(x_{2}\right)\left(x_{5,1}-\sum_{q} p_{q, 1}\left(x_{2}\right) x_{3, q}\right)^{r_{1}} \cdots\left(x_{5, k}-\sum_{q} p_{q, k}\left(x_{2}\right) x_{3, q}\right)^{r_{k}} .
$$

Переобозначим для краткости

$$
x_{2}=x, \quad x_{3}=y, \quad x_{5}=t, \quad \sum_{q} p_{q, j}(x) y_{q}=p^{j}(x, y)=\sum_{l, m} a_{l m}^{j} x_{l} y_{m} .
$$

Заметим, что $p^{j}(x, y)$ по условию оказьваются линейно независимы как многочлены от $x$ и $y$. Введем функцию

$$
\varphi(x, s)=\varphi\left(x, s_{1}, \ldots, s_{k}\right)=\sum_{r_{1}, \ldots, r_{k}} C_{r_{1}, \ldots, r_{k}} s_{1}^{r_{1}} \cdots s_{k}^{r_{k}}
$$

и векторные поля

$$
X_{j}(y)=\sum_{q} \frac{\partial p^{q}}{\partial x_{j}} \frac{\partial}{\partial s_{q}}=\sum_{q, m} a_{j m}^{q} y_{m} \frac{\partial}{\partial s_{q}}
$$

Теперь вспомним, что тейлоровское разложение $A$ не содержит $x_{2}$ в степени вьше первой. В новых обозначениях это условие запишется так:

$$
\varphi_{x_{1} x_{j}}(x, s)-X_{j} \varphi_{x_{i}}(x, s)-X_{i} \varphi_{x_{j}}(x, s)+X_{j} X_{i} \varphi(x, s)=0 .
$$

Выделим и приравняем к нулю член, свободньй относительно переменных $y$. Получим

$$
\varphi_{x_{1} x_{j}}(x, s)=0
$$


поэтому можно записать

$$
\varphi(x, s)=\varphi_{0}(s)+\sum_{q=1}^{k_{2}} x_{q} \varphi_{q}(s) .
$$

Теперь выделим и приравняем к нулю квадратичный по $y$ член. Получим

$$
X_{j} X_{i}\left(\varphi_{0}(s)+\sum_{q=1}^{k_{2}} x_{q} \varphi_{q}(s)\right)=0
$$

откуда

$$
\left.X_{j} X_{i} \varphi_{q}(s)\right)=0, \quad q=0,1, \ldots, k_{2} .
$$

Продифференцировав это равенство дважды по произвольньгм $y_{m}, y_{l}$, получим для любых $i, j, q, l, m$,что

$$
\left(\frac{\partial X_{j}}{\partial y_{m}} \frac{\partial X_{i}}{\partial y_{l}}+\frac{\partial X_{i}}{\partial y_{m}} \frac{\partial X_{j}}{\partial y_{l}}\right) \varphi_{q}=0
$$

Обозначим

$$
\frac{\partial X_{j}}{\partial y_{m}}=\sum_{q} a_{j m}^{q} \frac{\partial}{\partial s_{q}}=X_{j m}^{0} .
$$

Приняв $i=j, l=m$, получим

$$
\left(X_{j m}^{0}\right)^{2} \varphi_{q}(s)=0 .
$$

Рассмотрим систему векторных полей $X_{j q}^{0}$. Так как $p^{j}$ линейно независимы, ее ранг будет равен рангу системы полей $\partial / \partial s_{q}$. Выберем в линейной оболочке системы $\left\{X_{j q}^{0}\right\}_{j q}$ базис и соответствующие ему координатные функции $S_{j}$, линейно связанные с $s_{j}$. Получим

$$
\frac{\partial^{2} \varphi_{q}}{\partial S_{j}^{2}}=0
$$

откуда сразу следует, что все $\varphi_{q}$ являются многочленами степени не вьше $k$. Лемма доказана.

ЗАмечаниЕ 2.1. Данную в лемме оценку на степень $A\left(x_{2}, x_{3}, x_{5}\right)$ можно улучшить. Вспомним снова, что $A$ может зависеть от $x_{2}$ не более чем линейно. Значит, степенное разложение $A$ по $x_{2}, x_{3}, x_{5}$ не может содержать членов степени выше первой по $x_{2}$. В этом разложении присутствуют члены, полученные из произведений видов $x_{5}^{l}\left(p\left(x_{2}, x_{3}\right)\right)^{m}$ и $x_{2} x_{5}^{l}\left(p\left(x_{2}, x_{3}\right)\right)^{m}$, где $l+m \leqslant k$, т.е. мономы степени $l$ по $x_{5}$, степени $m$ по $x_{3}$, степеней $m$ и $m+1$ соответственно по $x_{2}$. Членов с $m>1$ быть не должно. Поэтому $\operatorname{deg} A \leqslant k+1$.

ЗАмЕчАниЕ 2.2. Из доказательства легко получить оценку вещественной размерности пространства решений выписанных уравнений для $A=\left(A_{1}, \ldots, A_{n}\right)$ (обозначим это пространство буквой $L)$ :

$$
\operatorname{dim} L \leqslant 2 n\left(k_{2}+1\right) 2^{k} .
$$

Вернемся к старым обозначениям.

Выпишем теперь соотношения $(3,2 \mid 2)$ и $(3,2 \mid 3)$ :

$$
\Delta_{32} G_{2}=0, \quad \Delta_{32} G_{3}=0 .
$$


Применим к ним $\Delta_{21}+\Delta_{212} ;$ применим $\Delta_{32}$ к $(2,1 \mid 2)$ и $(2,1 \mid 2)$. Получаем $\Delta_{32} B=0$, $\Delta_{32} C=0$. Вспомнив, что $\Delta_{212} B=0, \Delta_{212} C=0$, получаем, что $B$ и $C$ не зависят от переменных групшы $x_{5}$.

Итак, получили, что

$$
A=\varphi_{0}\left(x_{5}-p\left(x_{2}, x_{3}\right)\right)+\sum_{q=1}^{k} x_{2, k} \varphi_{q}\left(x_{5}-p\left(x_{2}, x_{3}\right)\right),
$$

$B$ и $C$ от $x$ не зависят.

Если вьписать теперь недостающие соотношения $(2,2 \mid 4),(2,2 \mid 5),(3,2 \mid 4),(3,2 \mid 5)$, все $G_{j}$ можно выразить через вещественньй постоянньй $\left(k_{2}+k_{3}+k_{4}+k\right)$-мерньй вектор $G_{0}, A, B$ и $C$.

Итак, вспомнив, что $B$ - комплексная матрица размера $n \times n$ и задается $2 n^{2}$ вешественньми параметрами, $C$ - квадратичная форма от $z$ со значениями в $\mathbb{C}^{n}\left(n^{2}(n+1)\right.$ вещественных параметров), а также оценки на размерность пространства решений для $A$ и значения $k_{2}, k_{3}, k_{4}$, мы можем получить следующие оценки.

ПРЕДЛОЖЕнИЕ 2.1. Пусть $Q$ - невырожденная модельная поверхность пятой степени. Тогда

$$
\begin{aligned}
\operatorname{dim} \text { aut } Q \leqslant & 2 n\left(n^{2}+1\right) 2^{k}+2 n^{2}+n^{2}(n+1) \\
& +\left(n^{2}+n^{2}(n+1)+\frac{1}{12} n^{2}(n+1)(n+2)(3 n+5)+k\right), \\
\operatorname{dim}_{\text {aut }_{0} Q \leqslant} & 2 n\left(n^{2}+1\right) 2^{k}-2 n+2 n^{2}+n^{2}(n+1) .
\end{aligned}
$$

(Параметры, определяющие эти алгебры, связаны соотношениями (3), обеспечивающими касание.)

ПредлоЖенИЕ 2.2. Степени инфинитезимальных автоморфизмов модельной поверхности не превышают $k+5$. Точнее, для инфинитезимального автоморфизма $\left(f, g_{2}, g_{3}, g_{4}, g_{5}\right)$ верны следуюшие оченки:

$\operatorname{deg} f \leqslant k+1, \quad \operatorname{deg} g_{2} \leqslant k+2, \quad \operatorname{deg} g_{3} \leqslant k+3, \quad \operatorname{deg} g_{4} \leqslant k+4, \quad \operatorname{deg} g_{5} \leqslant k+5$.

Итак, алгебра Ли aut $Q$ инфинитезимальных автоморфизмов поверхности $Q$ - это подалгебра алгебры полиномиальных векторных полей, чь степени ограничены некоторой константой, зависящей только от $n$ и $k$. Векторные поля, образуюшие aut $Q$ имеют вид $(2)$, где коэффициенты $\left(f, g_{2}, \ldots, g_{5}\right)$ голоморфны в окрестности нуля. Назначая веса следуюшим естественным образом:

$$
[z]=1, \quad\left[\frac{\partial}{\partial z}\right]=-1, \quad\left[w_{j}\right]=j, \quad\left[\frac{\partial}{\partial w_{j}}\right]=-j,
$$

сделаем aut $Q$ градуированной алгеброй Ли со следуюшим, непосредственно проверяемым свойством: если некоторое поле содержится в aut $Q$, то каждая его градуированная компонента - тоже. Можно написать, что

$$
\text { aut } Q=g_{-5}+\cdots+g_{-1}+g_{0}+g_{1}+\cdots+g_{D} .
$$


Подалгебре aut $+Q=g_{1}+\cdots+g_{D}$ соответствует подгруппа Aut $Q$ нелинейных преобразований $Q$, сохраняющих начало координат на месте. Подалгебре $g_{0}$ соответствует подгруппа LAut $Q$ линейных автоморфизмов $Q$, сохраняюших начало координат на месте. Эта подгруппа, в любом случае, содержит преобразования вида

$$
z \mapsto t z, \quad w_{j} \mapsto t^{j} w_{j}, \quad j=2, \ldots, 5, \quad t \in \mathbb{R}
$$

$g_{0}$, в свою очередь, всегда содержит соответствующий инфинитезимальньй автоморфизм вида $\left(z, 2 w_{2}, \ldots, 5 w_{5}\right)$. О подалгебре aut_ $Q=g_{-5}+\cdots+g_{-1}$ и соответствующей ей подгруппе еще вспомним ниже в п. 3.

3. Некоторые итоги. Покажем, что модельная поверхность обладает экстремальным свойством: размерность групшы голоморфных автоморфизмов невырожденного порождающего ростка вешественно-аналитического $C R$-многообразия не превосходит размерности группы его касательной модельной поверхности.

ПРЕДЛОЖЕНИЕ 3.1. Пусть $M_{0}$ - невырожденный порождающий росток ти$n a\left(n, k_{2}+k_{3}+k_{4}+k\right)$, a $Q$ - его касательная модельная поверхность. Тогда $\operatorname{dim}$ Aut $M_{0} \leqslant \operatorname{dim}$ Aut $Q$.

ДоКАЗАТЕЛЬСТВО вполне аналогично доказательству соответствующих оценок для квадрики и кубики (см., например, [7]) и представляет собой некоторую версию теоремы о неявной функции для формальных рядов с оценкой числа параметров. Показывается, что отображение

$$
f=z+f_{2}+\cdots, \quad g_{j}=w_{j}+g_{j, j+1}
$$

(здесь $f_{m}, g_{j, m}$ означает компоненту веса $m$ ) ростка

$$
\begin{aligned}
& y_{2}=F_{11}(z \bar{z})+\Phi_{2,3} \\
& y_{3}=2 \operatorname{Re} F_{21}\left(z^{2} \bar{z}\right)+\Phi_{3,4}, \\
& y_{4}=2 \operatorname{Re} F_{31}\left(z^{3} \bar{z}\right)+F_{22}\left(z^{2} \bar{z}^{2}\right)+\Phi_{4,5}, \\
& y_{5}=2 \operatorname{Re}\left(F_{41}\left(z^{4} \bar{z}\right)+F_{32}\left(z^{3} \bar{z}^{2}\right)+F_{212}\left(x_{2} z^{2} \bar{z}\right)\right)+\Phi_{5,6}
\end{aligned}
$$

в росток

$$
\begin{aligned}
& y_{2}=F_{11}(z \bar{z})+\widetilde{\Phi}_{2,3}, \\
& y_{3}=2 \operatorname{Re} F_{21}\left(z^{2} \bar{z}\right)+\widetilde{\Phi}_{3,4}, \\
& y_{4}=2 \operatorname{Re} F_{31}\left(z^{3} \bar{z}\right)+F_{22}\left(z^{2} \bar{z}^{2}\right)+\widetilde{\Phi}_{4,5}, \\
& y_{5}=2 \operatorname{Re}\left(F_{41}\left(z^{4} \bar{z}\right)+F_{32}\left(z^{3} \bar{z}^{2}\right)+F_{212}\left(x_{2} z^{2} \bar{z}\right)\right)+\widetilde{\Phi}_{5,6}
\end{aligned}
$$

задается не большим числом параметров,чем автоморфизм сооветствующей невырожденной модельной поверхности.

ПРЕДЛОЖЕНИЕ 3.2. Модельная поверхность однородна, т.е. голоморфные автоморфизмы действуют на ней транзитивно. 
ДокАЗАТЕЛЬСтво. За координаты на поверхности можно принять $\left(z, x_{2}, x_{3}, x_{4}, x_{5}\right)$. Надо проверить, что при произвольных $p \in \mathbb{C}^{n}, q \in \mathbb{R}^{k}$ точку с координатами $\left(z, x_{2}, x_{3}\right.$, $\left.x_{4}, x_{5}\right)$ биголоморфным преобразованием можно перевести в точку с координатами $(z+p$, $\left.x_{2}+q_{2}, x_{3}+q_{3}, x_{4}+q_{4}, x_{5}+q_{5}\right)$.

Сделаем подстановку $z \mapsto z+p, x \mapsto x+q$. В уравнениях поверхности тогда появятся члены меньшего веса. Теперь применим те же (полиномиальные) преобразования, что и в п. 1 при приведении уравнений поверхности к виду (1). Получим уравнения в прежнем виде.

Преобразования, упомянутые в доказательстве (назьваемые треугольно-полиномиальньпи), образуют в группе автоморфизмов $Q$ Aut $Q$ подгрупп, действующую на $Q$ транзитивно и без неподвижных точек. Эта подгруппа соответствует подалгебре aut_ $Q=g_{-5}+\cdots+g_{-1}$. Можно сформулировать следующее

ПРЕДЛОЖЕНИЕ 3.3. Модельная поверхность обладает естественной структурой группь Ли, действующей в $\mathbb{C}^{n+K}$ треугольно-полиномиальными преобразованиями.

ДокАЗАТЕЛЬСтво. Отождествление $Q \simeq$ Aut $-Q$ можно задать так: $\xi \mapsto \varphi_{\xi}$, где $\varphi_{\xi}$ - единственное преобразование из Aut_ $Q$, переводящее начало координат в точку $\xi$.

ПРЕДЛОЖЕНИЕ 3.4. Группа голоморфных преобразований невырожденной модельной поверхности Aut $Q$ является подгруппой группы бирачиональных преобразований $\mathbb{C}^{n+K}$, чьи степени не превосходят $(n+K+1)(k+5)+4$. Точнее, для произвольного автоморфизма $Q$ с компонентами $Z(z, w), W_{2}(z, w), W_{3}(z, w)$, $W_{4}(z, w), W_{5}(z, w)$ верны следующие оценки:

$$
\begin{aligned}
\operatorname{deg} Z & \leqslant(n+K+1)(k+5), \\
\operatorname{deg} W_{2} & \leqslant(n+K+1)(k+5)+1, \\
\operatorname{deg} W_{3} & \leqslant(n+K+1)(k+5)+2, \\
\operatorname{deg} W_{4} & \leqslant(n+K+1)(k+5)+3, \\
\operatorname{deg} W_{5} & \leqslant(n+K+1)(k+5)+4 .
\end{aligned}
$$

ДокАЗАТЕЛЬСТВо использует полученные в предложении 2.2 оценки на степени инфинитезимальных автоморфизмов, а также гарантированное наличие инфинитезимальных автоморфизмов из $g_{0}$ и aut_ $Q$. Ход рассуждений почти дословно повторяет ход доказательства бирациональности в [3] и [4]. Рассматривается действие группы Aut $Q$ на алгебре aut $Q$ и образы инфинитезимальных автоморфизмов из $g_{0}$ и aut_ $Q$ при этом действии. Так как эти образы также лежат в aut $Q$, и, следовательно, являются полиномиальными векторными полями ограниченной степени, из соответствуюших матричных соотношений получаются рациональность и оценки на степени производных

$$
\frac{\partial Z}{\partial z}, \frac{\partial W}{\partial w}, \frac{\partial Z}{\partial w}, \frac{\partial W}{\partial z}
$$

и самих $Z(z, w), W(z, w)$.

ПРЕДЛОЖЕНИЕ 3.5. Если две модельные поверхности биголоморфно әквивалентны, то они линейно әквивалентны. 
ДокАЗАТЕЛЬСтво проводится так же, как для квадрики и кубики (см., например, [7]). У отображающих функций выделяется линейная часть, которая сама осуществляет отбражение между модельными поверхностями.

Таким образом, можно видеть, что в нашей ситуации выполняются обычные свойства модельной поверхности. Тем самым основная теорема доказана.

\section{СПИСОК ЦИТИРОВАННОЙ ЛИТЕРАТУРЫ}

[1] Chern S.S., Moser J. K. Real hypersurfaces in complex manifolds // Acta Math. 1974. V. 133. №3-4. P. 219-271.

[2] Белошапка В.К.О голоморфных преобразованиях квадрики // Матем. сб. 1991. Т. 182. №2. C. 203-219.

[3] Туманов A.E. Конечномерность группы $C R$-автоморфизмов стандартного $C R$-многообразия и собственные голоморфные отображения областей Зигеля // Изв. АН СССР. Сер. матем. 1988. Т. 52. №3. С. 651-659.

[4] Белошапка В. К. Полиномиальные модели вещественных многообразий // Изв. РАН. Сер. матем. 2001. Т. 65. № 4. С. 3-20.

[5] Шананина Е. Н. Модели $C R$-многообразий типа $(1, K)$ при $3 \leqslant K \leqslant 7$ и их автоморфизмы // Матем. заметки. 2000. Т. 67. № 3. С. 452-459.

[6] Паламодов В. П. Линейные дифференциальные операторы с постоянными коэффициентами. М.: Наука, 1967.

[7] Белошапка В.К. Кубическая модель вешественного многообразия // Матем. заметки. 2001. T. 70. № 4. C. 503-519.

[8] Bloom T., Graham I. On type conditions for generic real submanifolds of $\mathbb{C}^{n} / /$ Invent. Math. 1977. V. 40. P. 217-243.

[9] Baouendi M.S., Ebenfelt P., Rothschild L.P. $C R$-authomorphisms of real analitic manifolds in complex space // Comm. Anal. Geom. 1998. V. 6. № 2. P. 291-315.

Московский государственный университет им. М.В. Ломоносова

Поступило 02.09.2003 